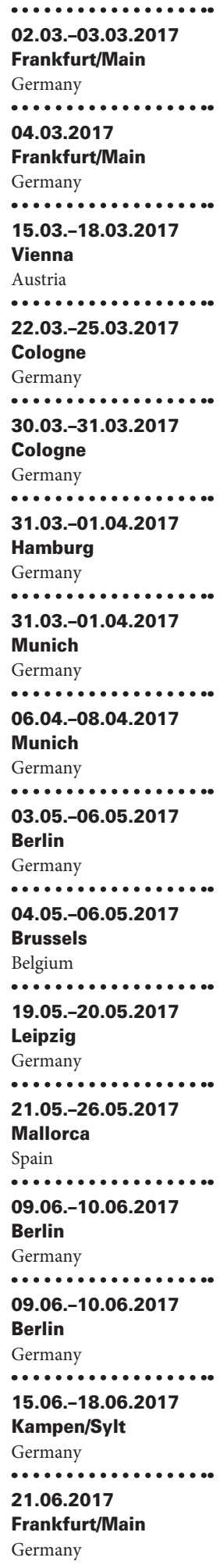

GBG Jahrestreffen 2017

[.................................................

AGO State of the Art Meeting 2017

15th St. Gallen International Breast Cancer Conference - Primary Therapy of Early Breast Cancer Evidence, Controversies, Consensus

Deutscher Schmerz- und Palliativtag - Praxis und Theorie der Versorgung

Master Class Breast Surgery

14. Hamburger Update Gynäkologie und Geburtshilfe

4. ASORS-Jahreskongress. Supportive Therapie und Rehabilitation bei Krebs - State of the Art 2017

AGO-Symposium. State of the Art Gynäkologische Onkologie

9. Internationale Charité-Mayo-Conference

\section{IMPAKT 2017 Breast Cancer Conference}

11. Jahrestagung der Mitteldeutschen Gesellschaft für Frauenheilkunde und Geburtshilfe

Senologie 2017 - 15. Internationaler Maritimer Kongress

5. BNGO-Hauptstadtkongress - Berufsverband Niedergelassener Gynäkologischer Onkologen

7. IQUO-Kongress - Interessenverband zur Qualitätssicherung der Arbeit niedergelassener Uro-Onkologen in Deutschland

6. Sylter Fortbildungstage Frauenheilkunde und Geburtshilfe

Neues aus Chicago
Information: www.gbg.de

Information: www.ago-online.de

Information: www.oncoconferences.ch/

Information: www.schmerz-und-palliativtag.de

Information: www.eickeler.org

Information: www.hamburger-updatede

Information:

www.kongresseonline.de/ASORS_2017/index.html

Information: www.if-kongress.de/veranstaltungen

Information: www.charite-mayo.de

Information:

www.esmo.org/Conferences/IMPAKT-2017-Breast-Cancer

Information: www.mgfg-kongress.de

Information: www.maritimerkongress.org

Information: www.bngo-kongress.de

Information: www.iquo-kongress.de

Information: www.gynsylt.de

Information: www.rhein-main-gyn.de

\begin{tabular}{ll}
\hline KARGER & ๑ 2017 S. Karger GmbH, Freiburg \\
Fax +497614520714 & Accessible online at: \\
$\begin{array}{l}\text { Information@Karger.com } \\
\text { www.karger.com }\end{array}$ & www.karger.com/brc
\end{tabular}




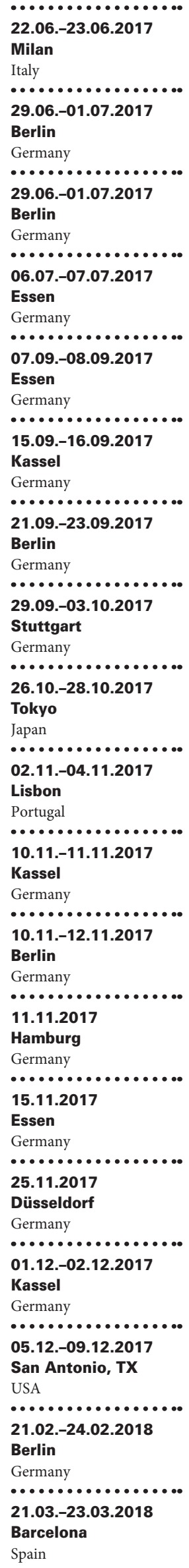

Milan Breast Cancer Biennial Conference 2017 - Innovation in Science and Care

37. Jahrestagung der Deutschen Gesellschaft für Senologie

Theoriemodule des Fortbildungsprogramms der Deutschen Akademie für Senologie

Master Class Breast Surgery

GynOnko live. Moderne operative Therapie - Multimodale Konzepte im Wandel

AGO-Zertifizierungskurs Onkologische Diagnostik und Therapie Bereich Gynäkologie

8. Kongress Forum Operative Gynäkologie

Jahrestagung der Deutschen, Österreichischen und Schweizerischen Gesellschaften für Hämatologie und Medizinische Onkologie

3rd World Congress on Controversies in Breast Cancer (CoBrCa)

Advanced Breast Cancer Fourth ESO-ESMO International Consensus Conference (ABC4)

AGO-Zertifizierungskurs Onkologische Diagnostik und Therapie Bereich Gynäkologie

8. Chefärzte-Jahrestagung der Bundesarbeitsgemeinschaft Leitender Ärztinnen und Ärzte in der Frauenheilkunde und Geburtshilfe e.V. (BLFG)

Hamburger Intensivseminar GynOnko

Best of Europe. State of the Art bei soliden Tumoren - Aktuelles vom Europäischen Krebskongress

7. Rhein-Ruhr-Symposium

[................................................ AGO-Zertifizierungskurs Onkologische Diagnostik und Therapie Bereich Gynäkologie

\section{San Antonio Breast Cancer Symposium}

33. Deutscher Krebskongress - Perspektiven verändern Krebs Krebs verändert Perspektiven. Diagnose, Therapie, (Über-)Leben

11th European Breast Cancer Conference (EBCC)
Information: http://breastmilan.com $/ \mathrm{mbcc} /$

Information: www.senologie.org

Information: www.akademie-senologie.de

Information: www.eickeler.org

Information: www.gyn-onko-live.de

Information: www.medconcept.org

Information: www.if-kongress.de/veranstaltungen

Information: www.haematologie-onkologie-2017.com

Information: http://cobrca.org

Information: www.abc-lisbon.org

Information: www.medconcept.org

Information: www.if-kongress.de/veranstaltungen

Information: www.eickeler.org/HIGO.pdf

Information: www.eickeler.org/BoE.pdf

Information: www.rhein-ruhr-symposium.de

Information: www.medconcept.org

Information: www.sabcs.org

Information:

www.krebsgesellschaft.de/deutsche-krebsgesellschaft/ konferenzen/deutscher-krebskongress.html

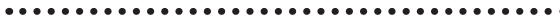

Information: www.ecco-org.eu/Events/EBCC11 